\title{
Oscilador Harmônico Fracionário via Transformada de Laplace
}

\author{
Daniela dos S. de Oliveira * E. Capelas de Oliveira \\ Departamento de Matemática Aplicada \\ Imecc - Unicamp \\ 13083-859, Campinas, SP \\ E-mail: ra142310@ime.unicamp.br, capelas@ime.unicamp.br
}

\section{RESUMO}

O cálculo de ordem não inteira, popularmente conhecido como cálculo fracionário, pode ser visto como uma generalização da diferenciação e integração usuais, isto é, a passagem de ordem inteira para ordem não inteira, ou mesmo complexa.

Este trabalho tem por objetivo discutir e resolver uma equação diferencial fracionária, em particular, a equação diferencial que generaliza o problema do oscilador harmônico clássico.

\section{Derivada fracionária segundo Caputo}

A equação diferencial fracionária do oscilador harmônico é, [4]

$$
\frac{d^{\alpha} x(t)}{d t^{\alpha}}+\omega^{2} x(t)=0
$$

com $\omega^{2}=\frac{k}{m}$, onde $k$ é uma constante, $m$ a massa, $\alpha$ um parâmetro e satisfazendo as seguintes condições iniciais

$$
x(0)=x_{0}, \quad x^{\prime}(0)=0 .
$$

Consideramos o parâmetro $1<\alpha \leq 2$, de modo que, no caso em que $\alpha=2$ recuperamos a solução do oscilador harmônico clássico. A derivada de ordem $\alpha$ é uma derivada no sentido de Caputo, [1].

Definição 1.1. A derivada fracionária de ordem $\alpha \in \mathbb{C}$, no sentido de Caputo, à esquerda, é definida por

$$
{ }_{a}^{C} D_{t}^{\alpha} f(t)={ }_{a} J_{t}^{n-\alpha} D^{n} f(t)
$$

com $n=[\operatorname{Re}(\alpha)]+1$, onde $[\operatorname{Re}(\alpha)]$ significa a parte inteira de $\operatorname{Re}(\alpha)$, ou seja,

$$
{ }_{a}^{C} D_{t}^{\alpha} f(t)={ }_{*} D_{a}^{\alpha} f(t)=\frac{1}{\Gamma(n-\alpha)} \int_{a}^{t} \frac{D^{n}[f(u)]}{(t-u)^{\alpha-n+1}} d u, \quad \operatorname{Re}(\alpha)>0, \mathrm{a} \in \mathbb{R}
$$

onde $D^{n}[f(u)]=\frac{d^{n} f(u)}{d u^{n}}$ é a derivada $n$ de ordem inteira e $J^{n-\alpha}$ é a integral de ordem fracionária.

\section{Funções de Mittag-Leffler}

Assim como a função exponencial é solução de equações diferenciais com coeficientes constantes, a função de Mittag-Leffler, por ser uma generalização da função exponencial, é solução de equações diferenciais fracionárias com coeficientes constantes. A função de Mittag-Leffler de um parâmetro é definida da seguinte maneira, $[2,3]$.

${ }^{*}$ Bolsista de Mestrado CAPES. 
Definição 2.1. A função de Mittag-Leffler de um parâmetro, denotada por $E_{\alpha}(z)$, conforme introduzida por Mittag-Leffler é uma função complexa que depende de um parâmetro complexo, $\alpha$, onde $\operatorname{Re}(\alpha)>0$, na forma de uma série

$$
E_{\alpha}(z)=\sum_{k=0}^{\infty} \frac{z^{k}}{\Gamma(k \alpha+1)}
$$

\section{Oscilador harmônico fracionário}

Aplicando a transformada de Laplace na equação diferencial fracionária do oscilador harmônico e utilizando as condições iniciais, temos que a solução é dada por

$$
x(t)=x_{0} E_{\alpha}\left(-\omega^{2} t^{\alpha}\right)
$$

cuja representação gráfica é, para diferentes valores do parâmetro $\alpha$

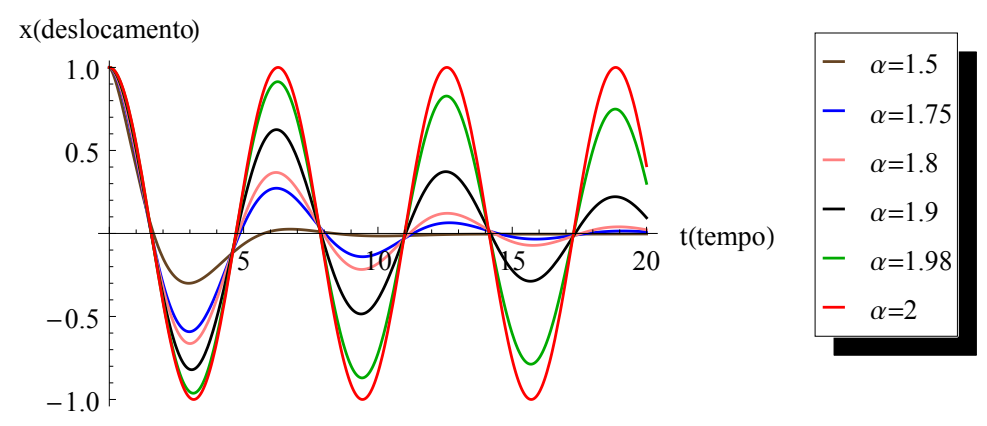

\section{Conclusão}

Utilizando a metodologia da transformada de Laplace resolveu-se a equação diferencial fracionária que descreve o problema do oscilador harmônico fracionário, uma generalização do caso clássico. Para melhor visualização da solução esboçamos um gráfico para distintos valores do parâmetro associado à ordem da derivada. No particular caso em que este parâmetro é igual a 2 recuperamos o caso do oscilador harmônico clássico.

Palavras-chave: Cálculo fracionário, Derivada de Caputo, Oscilador harmônico fracionário

\section{Referências}

[1] R. F. Camargo, E. Capelas de Oliveira, A. O. Chiacchio, "Sobre a Função de Mittag-Leffler", Relatório de Pesquisa 15/06, IMECC-Unicamp, 2006.

[2] M. G. Mittag-Leffler, "Sur la Nouvelle Fonction $E_{\alpha}(x)$ ”, C. R. Acad. Sci., 137, 554-558, 1903.

[3] D. S. Oliveira, "Derivada Fracionária e as Funções de Mittag-Leffler", Dissertação de Mestrado, IMECC-Unicamp, julho de 2014.

[4] D. C. Rosendo, "Sobre a Função de Mittag-Leffler", Dissertação de Mestrado, IMECC-Unicamp, 2008. 